\title{
Linearly Polarized Fed Circularly Polarized DRA Reflectarray
}

\author{
Abd-Elhady. M. A ${ }^{1, *}$, S. H. Zainud-Deen ${ }^{2}$, A. A. Mitkees ${ }^{3}$, A. A. Kishk ${ }^{4}$ \\ ${ }^{1}$ Faculty of Engineering, Benha University, Egypt \\ ${ }^{2}$ Faculty of Electronic Engineering, Elmonofia University, Egypt \\ ${ }^{3}$ Military Technical College, Egypt \\ ${ }^{4}$ Department of Electrical Engineering, University of Mississippi, USA
}

\begin{abstract}
Two dielectric resonator antennas are discussed to generate circularly polarized waves in a reflectarray antenna . In this case, two models are discussed here, one is DRA supported on cross slot, while the second is aperture coupled DRA supported on cross strip line coupled to DRA by cross slot. The verification of reflection coefficient phase of cell is satisfied by CST microwave studio and HFSS package. A complete array of $15 \times 15$ fed by a circular horn tilted by 45 degree was designed and simulated at $\mathrm{X}$-band by full wave package. The simulated results show that the obtained 1.5-dB gain bandwidth and 3-dB axial ratio bandwidth of the reflectarray with aperture coupled DRA can reach as large as $25 \%$ and $12.5 \%$.
\end{abstract}

Keywords Circular Polarization, Dielectric Resonator Antenna, Aperture Coupled DRA, Reflectarray

\section{Introduction}

High gain antennas with light weight and compactness are desired in satellite communications and radar applications. Downsizing of antennas and associated technologies have recently become somewhat of a trend in the antenna community. Reflectarrays offer a competitive alternative to reflectors in many narrow-band pencil-beam and shapedbeam antenna applications. One way to increase the antenna gain is to construct an antenna array with hundreds or thousands of elements. However, it usually suffers from a noticeable power loss in the feeding network. In contrast, a large aperture reflector antenna that avoids the usage of a feeding network can achieve a higher efficiency and hence is widely used in many communication systems. The reflectarray works much like a traditional reflector antenna. The planar reflectarray is rapidly becoming an attractive alternative because of its advantages such as low mass and volume, ease of manufacture, and possibilities offered for beam shaping and electronic beam control[1]. The circularly polarized (CP) antenna has been widely used since the electromagnetic wave emitted from the antenna is robust against the environmental interference. Therefore, a variety of different $\mathrm{CP}$ reflectarrays have been proposed and implemented in the literature. In[2,3], an angular rotation technique has been applied to the elements to attain the

* Corresponding author:

abdoeng78@gmail.com (M. A. Abd-Elhady)

Published online at http://journal.sapub.org/ijea

Copyright (C) 2012 Scientific \& Academic Publishing. All Rights Reserved phase delay for the CP reflectarrays. One essential condition of this technique is that the primary source must be a CP feed antenna. In[4],a linearly polarized feeder has been used with tilted angle of $45^{\circ}$ with variable cross slot patch reflectarray and cross strip patch reflectarray for generating circularly polarized reflected beam while in[5] both the dimensions of the patch element can be adjusted to achieve the required $90^{\circ}$ phase difference between orthogonal field components for circular polarization . Dielectric resonator antennas have been shown to offer several advantages including wide bandwidth and high radiation efficiency. They are viable candidates for numerous applications as either individual elements or in an array environment. A reflectarray that uses a DRA as the radiating element has been developed[6-10]. Also, DRA reflectarrays using linearly polarized feed was designed to realize circular polarization[7] based on the following principle. The polarization of the feed runs parallel to the diagonal line of the square cell element, and its linearly polarized field is decomposed into two equal components along the sides of the square cell. Then, the field with necessary phase shift on each component is reradiated by the cell element to attain a CP focused beam. In this letter, we present a DRA reflectarray cell element for designing the CP reflectarray antenna with linearly polarized feed. The reflectarray is illuminated by linearly polarized wave generated from a circular horn. A circularly polarized reflectarray using crossed slot DRA elements and cross strip line aperture coupled DRA are presented. The circular polarization is generated when a $90^{\circ}$ for RHCP or $-90^{\circ}$ for LHCP phase difference between the phase response of the two arms of the crossed slot DRA element or cross 
strip line aperture coupled DRA is achieved. The analyses are carried out using CST microwave studio[12]. The circularly polarized reflectarray is designed to operate at $\mathrm{X}$-band.

\section{Numerical Results}

\subsection{Circular Horn Feeder}

A center feed structure is used with a distance to the edge of the aperture of $10.5 \mathrm{~cm}$. A X-band circular polarized horn antenna with dual feeds probes shifted by $90^{\circ}$ for right hand circular polarization at $10 \mathrm{GHz}$ was used as feeder with center fed as shown in Figure. 4. The outer aperture of horn is $40 \mathrm{~mm}$, length of $40 \mathrm{~mm}$ while the waveguide of $20 \mathrm{~mm}$. The focal to reflectarray dimension is $\mathrm{F} / \mathrm{D}=1$ for both designs.

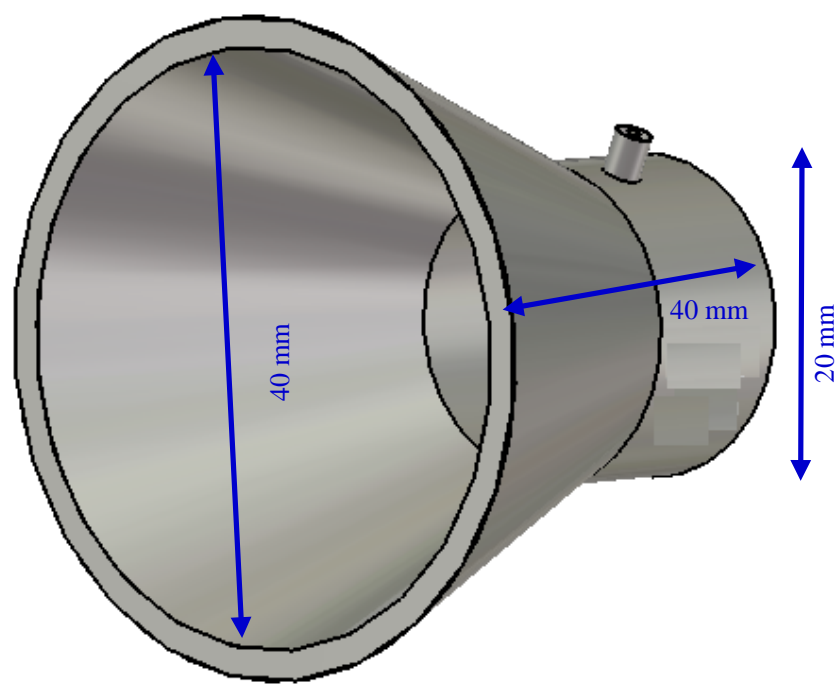

Figure 1. Circular horn structure.

Considering the array on the $x-y$ plane illuminated by a feed horn, the required phase distribution, $\Phi\left(x_{i}, y_{i}\right)$, at each element of the array to collimate a beam in the $\left(\theta_{o}, \varphi_{o}\right)$ direction is determined as:

$$
\begin{aligned}
& \varphi\left(x_{i}, y_{i}\right)=k_{o}\left(d_{i}-\sin \theta_{o}\left(x_{i} \cos \phi_{o}+y_{i} \sin \phi_{o}\right)\right) \\
& d_{i}=\sqrt{\left(x_{i}-x_{f}\right)^{2}+\left(y_{i}-y_{f}\right)^{2}+\left(z_{f}\right)^{2}}
\end{aligned}
$$

where $k_{o}$ is the propagation constant in vacuum, $d_{i}$ is the distance from the feed horn to the element $i$ of the array and $\left(x_{i}, y_{i}\right)$ are the coordinates of the element.

\subsection{DRA Supported on Variable Cross Slot Lengths}

The cell consists of DRA with fixed size with substrate of same dielectric constant of DRA of 10.2, supported on ground plane with variable slot lengths L1 and L2 with phase differ of 90 degree to generate RHCP reflected beam as shown in Figure. 2.

The fixed size DRA with cross slot cell is designed to generate circularly polarized reflected beam at X-band. The geometry of reflectarray cell structure dimension is shown in Figure. 2. The cell size is $11 \mathrm{~mm} \times 11 \mathrm{~mm}$ with dielectric resonator of fixed thickness of $2.5 \mathrm{~mm}$ with dielectric constant 10.2 supported on substrate with same dielectric constant with thickness of $0.5 \mathrm{~mm}$, ground with slot width $1.4 \mathrm{~mm}$, and variable length, Spacer of $2 \mathrm{~mm}$ and ground plane. The waveguide model is used to calculate the reflected wave phase versus the slot length variation. CST microwave studio and HFSS software[11] are used to investigate the performance of the antenna for the variation of the phase of the reflection coefficient against slot length $(\mathrm{L})$ with a phase range $\left(0^{\circ}-330^{\circ}\right)$ as shown in Figure. 3. Once the desired cell phase is calculated to compensate the different paths from feeder to reflectarray and make all reflected beams are coherently phase at the aperture plane by equation (1), L1 is calculated and L2 is calculated from the same Figure. 3 after add 90o to phase of L1.

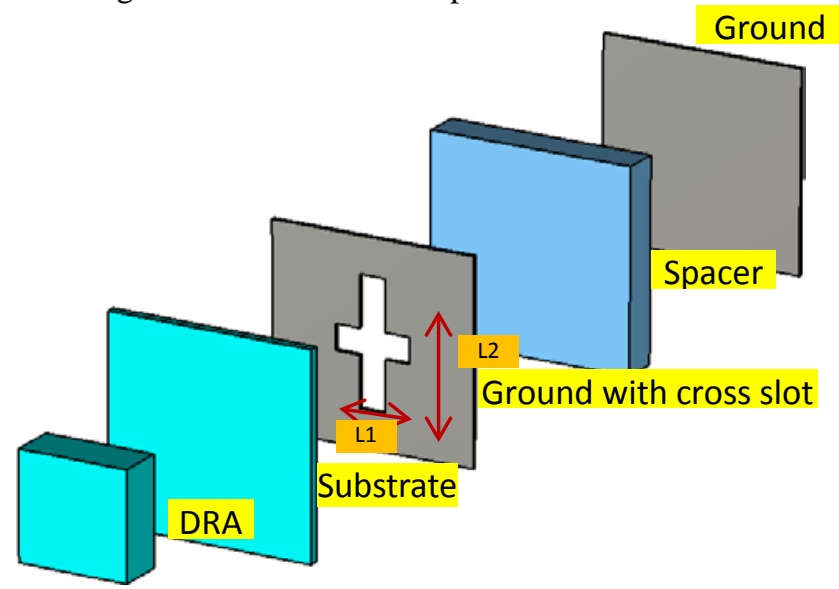

Figure 2. DRA with cross slot cell.

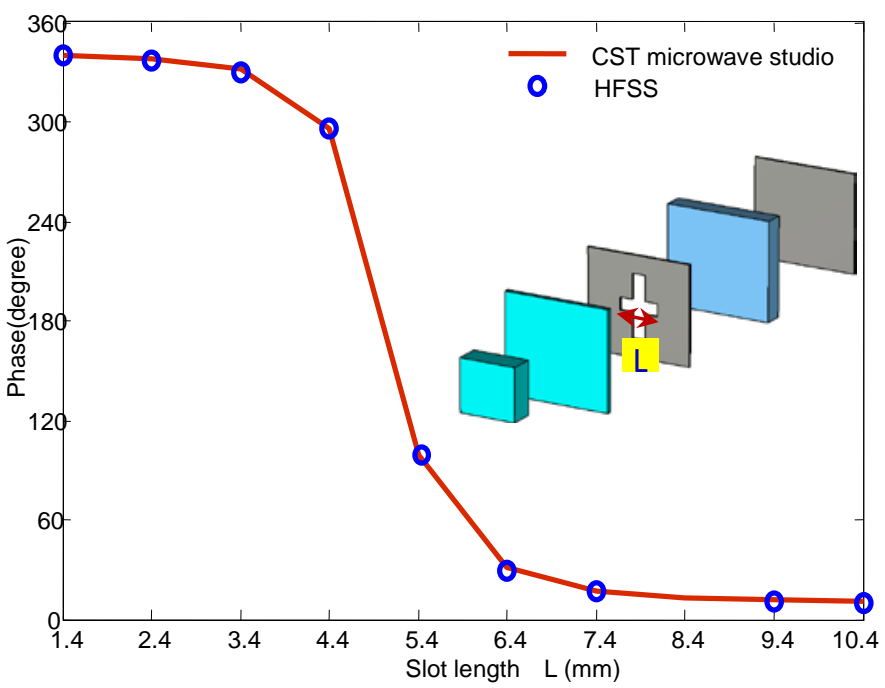

Figure 3. Phase versus slot length.

The complete array structure is shown in Figure. 4,with layers of cross slot of variable lengths. It subdivided into 225 elements of equal size of $11 \mathrm{~mm} \times 11 \mathrm{~mm}$ arranged in a lattice of 15 x 15 elements as shown in Figure. 4. Simulated $\mathrm{XZ}$ plane ( $\mathrm{H}$ plane) and $\mathrm{YZ}$ plane (E plane) are shown in Figure. 5 and 6 respectively. The reflected beam is broadside. As designed, the RHCP pattern is dominant and it achieves a maximum directivity of $24 \mathrm{~dB}$ at $10.5 \mathrm{GHz}$, the 
axial ratio at the desired frequency $10 \mathrm{GHz}$ was $2 \mathrm{~dB}$ with gain of $23.5 \mathrm{~dB}$. The $1.5 \mathrm{~dB}$ gain bandwidth of reflectarray is about $14 \%$ from $9.6 \mathrm{GHz}$ to $11 \mathrm{GHz}$ with aperture efficiency of $59 \%$ at $10 \mathrm{GHz}$ according to equation :

$$
G_{o}=\frac{4 \times \pi \times \eta_{a p} A_{\text {phy }}}{\lambda_{o}^{2}}
$$

Where $G_{o}$ is the gain, $A_{\text {phy }}$ is the physical area $(18 \mathrm{~cm} \mathrm{x}$ $18 \mathrm{~cm}$ ), While the $3 \mathrm{~dB}$ axial ratio bandwidth is from $9.8 \mathrm{GHz}$ to $10.9 \mathrm{GHz}$, about $11 \%$ as shown in Figure. 7 and 8 respectively.

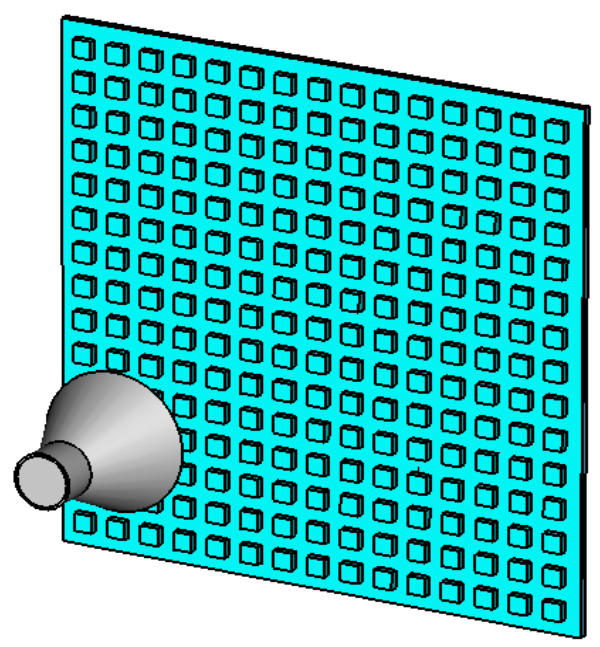

(a)

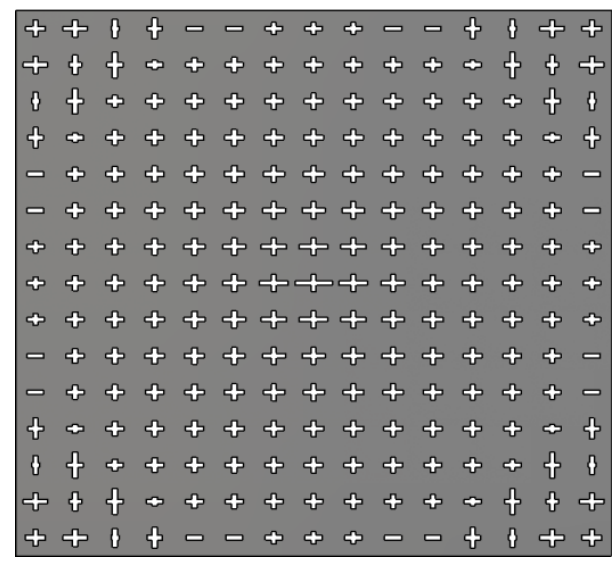

(b)

Figure 4. Array geometry a) 3 D view b)Cross slot layer.

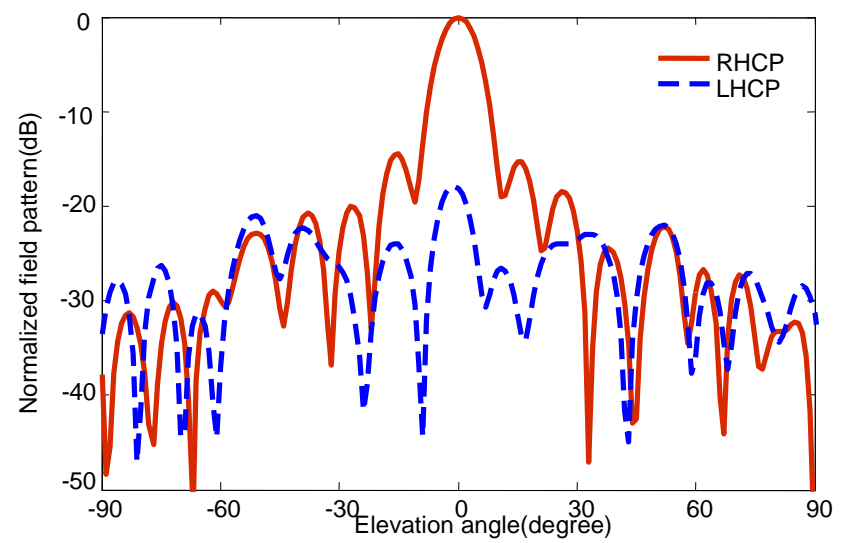

Figure 5. XZ plane normalized field pattern at $10 \mathrm{GHz}$.

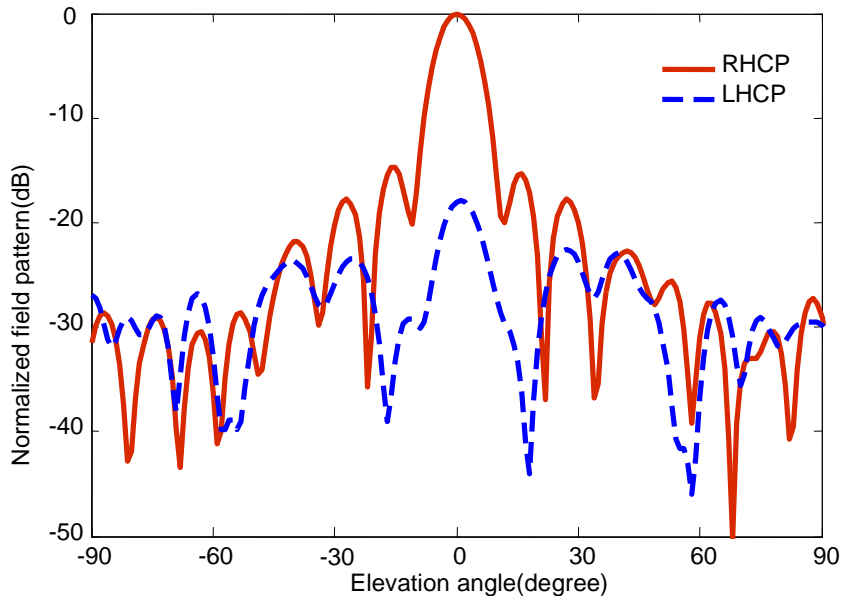

Figure 6. YZ plane normalized field pattern at $10 \mathrm{GHz}$.

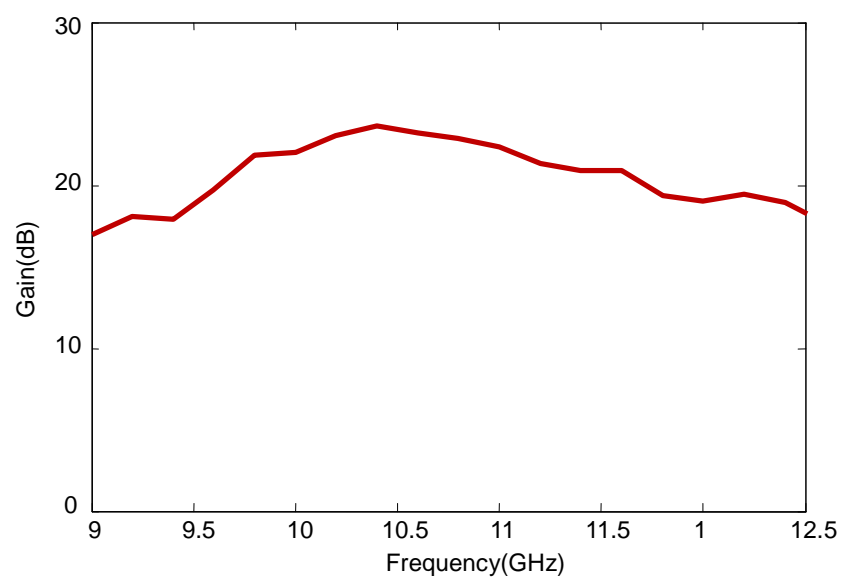

Figure 7. Gain versus frequency.

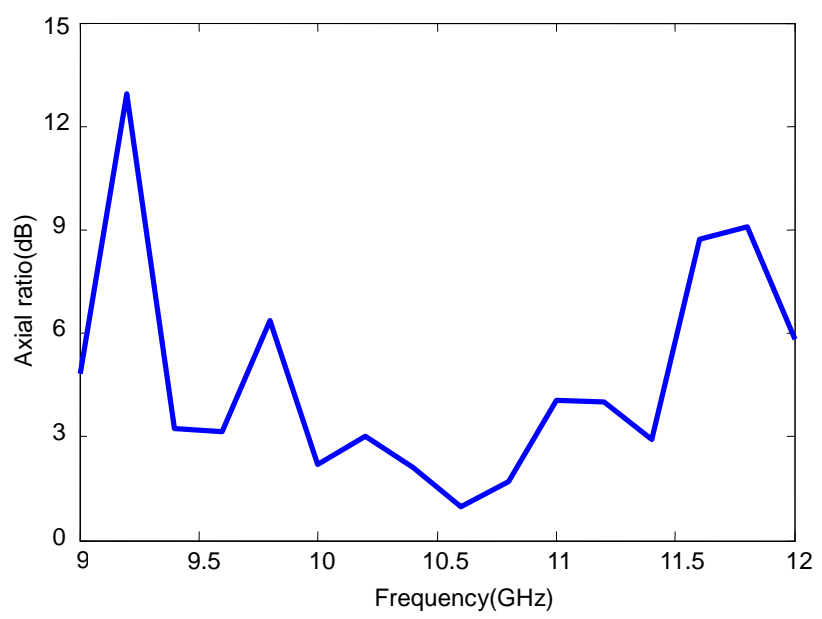

Figure 8. Axial ratio versus frequency.

\subsection{Aperture Coupled Reflectarray with Cross Strip} Lines

The cell is designed to operated at X-band with a phase range that covers 360 degree. The geometry of a aperture coupled DRA reflectarray cell structure dimensions is shown in Figure. 9.

The cell size is $12 \mathrm{~mm} \times 12 \mathrm{~mm}$ with dielectric resonator of fixed thickness of $6 \mathrm{~mm}$ with dielectric constant 10.2, ground with slot of dimension $5 \mathrm{~mm} \times 0.6 \mathrm{~mm}$, transmission 
line strip width of $0.8 \mathrm{~mm}$ with variable length was supported on lower substrate of dielectric constant 10.2, Spacer of $2.5 \mathrm{~mm}$ and ground plane. Also, the waveguide model is used to calculate the reflected wave phase versus the dielectric resonator sizes variation. CST microwave studio and HFSS software are used to investigate the performance of the antenna for the variation of the phase of the reflection coefficient against transmission line length (L) with a complete phase period $\left(0^{\circ}-360^{\circ}\right)$ as shown in Figure. 10.

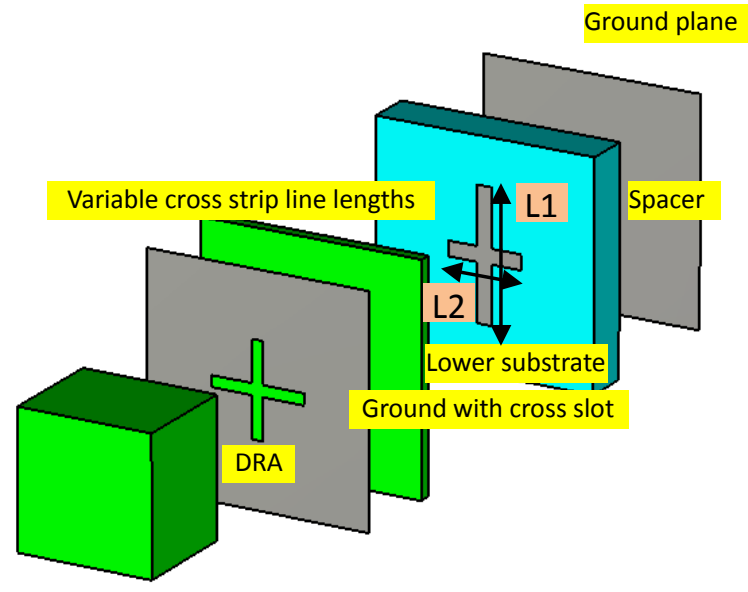

Figure 9. Aperture coupled DRA cell.

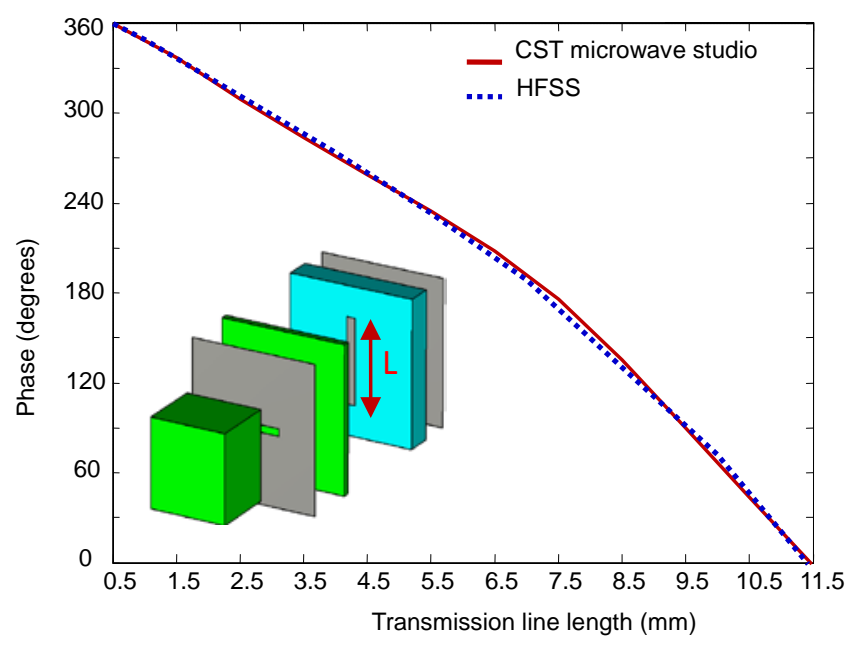

Figure 10. Phase versus DRA length.

The array is subdivided into 225 elements of equal size of $12 \mathrm{~mm} \times 12 \mathrm{~mm}$ arranged in a lattice of $15 \times 15$ elements. Simulated XZ plane(H plane) and YZ plane(E plane) are shown in Figure. 11 and 12 respectively. Also as designed, the RHCP pattern is dominant and it achieves a maximum directivity of $23.5 \mathrm{~dB}$ at $10.5 \mathrm{GHz}$, the axial ratio at the desired frequency $10 \mathrm{GHz}$ was $1 \mathrm{~dB}$ with gain of $23 \mathrm{~dB}$.The $1.5 \mathrm{~dB}$ gain bandwidth of reflectarray is about $25 \%$ from $9.5 \mathrm{GHz}$ to $12 \mathrm{GHz}$ while the $3 \mathrm{~dB}$ axial ratio is from $9 \mathrm{GHz}$ to $10.4 \mathrm{GHz}$ or about $14 \%$ with aperture efficiency of $44 \%$ at $10 \mathrm{GHz}$ as shown in Figure. 13 and Figure. 14,respectively. Also, as the desired cell phase is calculated to compensate the different paths from feeder to reflectarray and make all reflected beams are coherently phase at the aperture plane by equation (1), L1 is calculated and L2 is calculated from the same Figure. 10 after add $90^{\circ}$ to phase of L1.

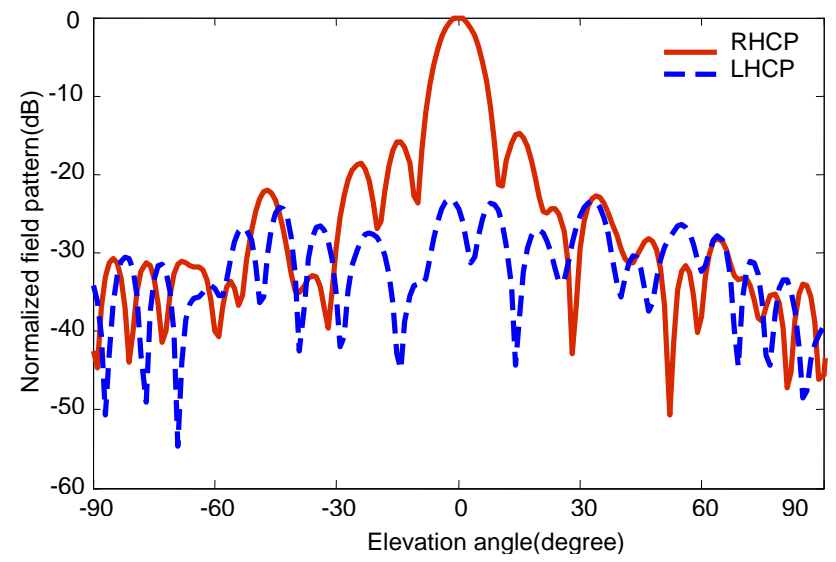

Figure 11. YZ plane normalized pattern at $10 \mathrm{GHZ}$

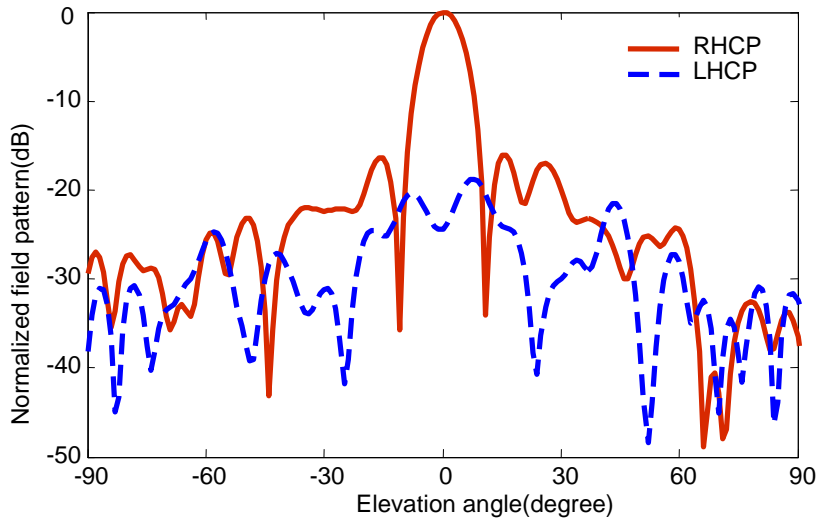

Figure 12. $\mathrm{XZ}$ plane normalized pattern at $10 \mathrm{GHZ}$.

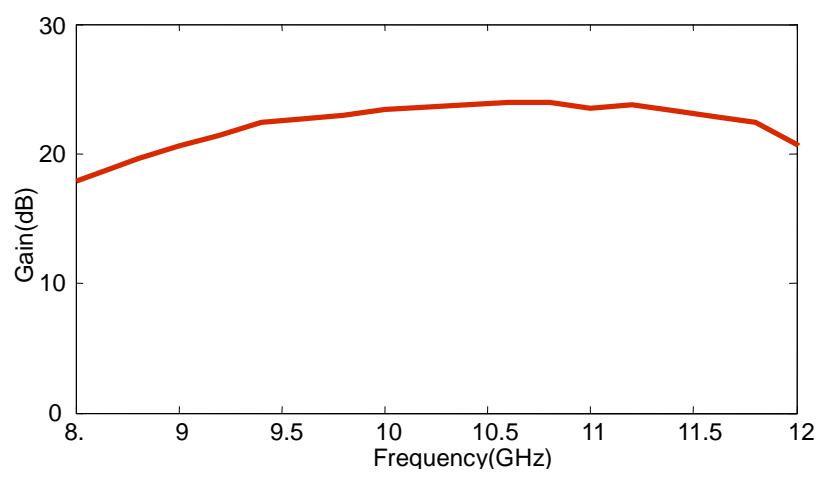

Figure 13. Gain versus frequency.

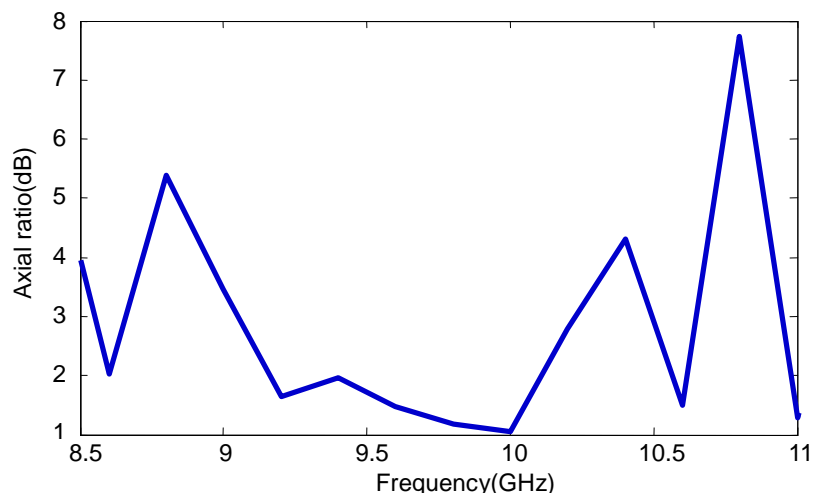

Figure 14. Axial ratio versus frequency. 


\section{Conclusions}

New types of designing circularly polarized reflectarray antenna was presented in this paper. DRA with cross slot of variable slot lengths is presented with suitable gain of $23.5 \mathrm{~dB}$ at $1 \mathrm{GHz}$ and $3 \mathrm{~dB}$ axial ratio of $11 \%$ at $\mathrm{X}$-band. The second model was aperture coupled DRA with cross strip line and cross slot are presented with gain of $23 \mathrm{~dB}$ at $10 \mathrm{GHz}$ and $3 \mathrm{~dB}$ axial ratio of $14 \%$ at $\mathrm{X}$-band. The phase figure was verified using CST and HFSS with good agreement between them.

\section{REFERENCES}

[1] J. Huang and J. A. Encinar, Reflectarray Antennas, John Wiley \& Sons Inc., Hoboken, NJ, 2007

[2] A. Yu, F. Yang, Elsherbeni, A. Z., huang, J.;" Design and measurement of a circularly polarized Ka-band reflectarray antenna "; Antennas and Propagation, 2009. EuCAP 2009. 3rd European Conference; 2009, Page(s): 2769 - 2773

[3] Han, C.; Huang, J.; Kai Chang; "Cassegrain Offset Subreflector-Fed X/Ka Dual-Band Reflectarray With Thin Membranes"; IEEE Trans. Antennas Propagat.,Volume: 54 , Issue: 10 ; 2006, Page(s): 2838 - 2844

[4] M. R. Chaharmir, J. Shaker, M. Cuhaci and A. Sebak "Circularly polarised reflectarray with cross-slot of varying arms on ground plane" Electron. Lett. 2002, Vol. 38 No. 24

[5] M. G. Keller, J. Shaker and A. Petosa, A. Ittipiboon, M. Cu- haci, Y. M. M. Antar, "A Ka-band dielectric resonator antenna reflectarray", European Microwave Conference 2000, Paris, France, pp. 272-275, Oct. 2000

[6] G. Zhao; Y. C. Jiao; F. Zhang; F. S. Zhang; "A subwavelength element for broadband circularly polarized reflectarrays "IEEE Antennas and Wireless propagate. Lett. VOL. 9, 2010

[7] S. H. Zainud-Deen; A. M. Abd-Elhady; A. A. Mitkees; A. A. Kishk; "Design of Dielectric Resonator Reflectarray Using Full-Wave Analysis," 26th National Radio Science Conference (NRSC 2009), Faculty of Engineering, Future Univ., Egypt, B10, pp. 1-9, March 2009

[8] S. H. Zainud-Deen; A. M. Abd-Elhady; A. A. Mitkees; A. A. Kishk; "Dielectric Resonator Reflectarray with Two DRA Sizes and Varying Slot Loading," 2010 IEEE AP-S International Symposium on Antennas and Propagation and USNC/URSI National Radio Science Meeting, Toronto, Canada, 2010

[9] A. M. Abd-Elhady; S. H. Zainud-Deen; A. A. Mitkees; A. A. Kishk;"Slot-Loading Rectangular Dielectric Resonator Elements Reflectarray",1st Middle East Conference on Antennas and Propagation, (MECAP), Cairo, Egypt, Oct. 2010

[10] A. M. Abd-Elhady; S. H. Zainud-Deen; A. A. Mitkees; A. A. Kishk;"X-Band Linear Polarized Aperture-Coupled DRA Reflectarray," 2010 International Conference on Microwave and Millimeter Wave Technology, Chengdu, China, 2010

[11] HFSS: High frequency structure simulator based on finite element method, v11.0.2, Ansoft Corporation, 2007

[12] CST microwave studio,www.cst.com 